\title{
Estimación poblacional y conservación de felinos (Carnivora: Felidae) en el norte de Quintana Roo, México
}

\author{
Dulce María Ávila-Nájera*1, Cuauhtémoc Chávez², Marco A. Lazcano-Barrero³, Sergio Pérez- \\ Elizalde $^{4} \&$ José Luis Alcántara-Carbajal ${ }^{5}$ \\ 1. Colegio de Postgraduados, Campus Montecillo, Carretera México-Texcoco, km. 36.5 C.P. 56230. Montecillo, Mpio. \\ de Texcoco, México; dul.avna@gmail.com \\ 2. Departamento de Ciencias Ambientales, CBS Universidad Autónoma Metropolitana, Unidad Lerma, Hidalgo Pte. 46, \\ Col. La Estación Lerma, Estado de México C.P. 52006, México; j.chavez@correo.ler.uam.mx \\ 3. Reserva Ecológica El Edén A. C, Teocaltiche 207, SM 45, M4, L3, Fracc. Villas del Sol, Cancún Quintana Roo C.P. \\ 77506; mlazcanobarrero@hotmail.com \\ 4. Colegio de Postgraduados, Campus Montecillo, Carretera México-Texcoco, km. 36.5 C.P. 56230. Montecillo, Mpio. \\ de Texcoco, México; sergiop@colpos.mx \\ 5. Colegio de Postgraduados, Campus Montecillo, Carretera México-Texcoco, km. 36.5 C.P. 56230. Montecillo, Mpio. \\ de Texcoco, México; jlalcant@colpos.mx \\ * Correspondence
}

Recibido 30-VII-2014. Corregido 25-III-2015. Aceptado 28-IV-2015.

\begin{abstract}
Population estimates and conservation of felids (Carnivora: Felidae) in Northern Quintana Roo, Mexico. Wildlife density estimates provide an idea of the current state of populations, and in some cases, reflect the conservation status of ecosystems, essential aspects for effective management actions. In Mexico, several regions have been identified as high priority areas for the conservation of species that have some level of risk, like the Yucatan Peninsula (YP), where the country has the largest population of jaguars. However, little is known about the current status of threatened and endangered felids, which coexist in the Northeastern portion of the Peninsula. Our objective was to estimate the wild cats' density population over time at El Eden Ecological Reserve (EEER) and its surrounding areas. Camera trap surveys over four years (2008, 2010, 2011 and 2012) were conducted, and data were obtained with the use of capture-recapture models for closed populations (CAPTURE + MMDM or $1 \frac{2}{2}$ MMDM), and the spatially explicit capture-recapture model (SPACECAP). The species studied were jaguar (Panthera onca), puma (Puma concolor), ocelot (Leopardus pardalis), jaguarundi (Puma yaguaroundi) and margay (Leopardus wiedii). Capture frequency was obtained for all five species and the density for three (individuals $/ 100 \mathrm{~km}^{2}$ ). The density estimated with The Mean Maximum Distance Moved (MMDM), CAPTURE, ranged from 1.2 to 2.6 for jaguars, from 1.7 to 4.3 for pumas and from 1.4 to 13.8 for ocelots. The density estimates in SPACECAP ranged from 0.7 to 3.6 for jaguars, from 1.8 to 5.2 for pumas and 2.1 to 5.1 for ocelots. Spatially explicit capture recapture (SECR) methods in SPACECAP were less likely to overestimate densities, making it a useful tool in the planning and decision making process for the conservation of these species. The Northeastern portion of the Yucatan Peninsula maintains high populations of cats, the EEER and its surrounding areas are valuable sites for the conservation of this group of predators. Rev. Biol. Trop. 63 (3): 799-813. Epub 2015 September 01.
\end{abstract}

Key words: keystone species, priority areas for conservation, density, abundance, Leopardus, Panthera, Puma.

La constante presión antropogénica sobre el ambiente ha provocado que la diversidad biológica se encuentre globalmente amenazada y muchas especies se hallen al borde de la extinción (Sarukhán, Carabias, Koleff, \&
Urquiza-Haas, 2012; Dirzo et al., 2014; Pimm et al., 2014), como los grandes depredadores (Hoogesteijn, Hoogesteijn, \& Mondolfi, 1993; Amit, Gordillo-Chávez \& Bone, 2013), los cuales juegan un papel fundamental en el 
funcionamiento y equilibrio del ecosistema (Beschta \& Ripple, 2009; Estes et al., 2011).

México es un ejemplo claro del deterioro que han sufrido las poblaciones de grandes depredadores, cuatro de las especies de felinos silvestres que habitan sus zonas tropicales se encuentran en alguna categoría de riesgo. El jaguar (Panthera onca), tigrillo (Leopardus wiedii) y jaguarundi (Puma yaguaroundi) estan en el apéndice I y el ocelote (Leopardus pardalis) en el apéndice II de CITES. Estas cuatro especies también mantienen en la legislación mexicana el estatus de especies en peligro de extinción o amenazadas, y su cacería esta prohibida. El puma (Puma concolor), a pesar de tener una distribución más amplia, es considerado una especie que requiere de protección especial (Sedesol, 1994; Semarnat, 2010).

La Península de Yucatán es una zona de gran importancia para la conservación de estos depredadores ápice, específicamente del jaguar, al encontrarse cerca de 2000 jaguares, la mitad de lo que se ha estimado para México (Ceballos, Chávez, \& Zarza, 2011) y por mantener la mayor porción de selvas altas y medianas de la entidad (Sanderson et al., 2002; Ceballos, Chávez, Zarza, \& Amín, 2005; Manterola et al., 2011). Además, el jaguar es una especie considerada, sombrilla, clave e indicadora, que junto con las otras especies de felinos con las cuales cohabita, contribuye a mantener el equilibrio de los ecosistemas (Miller \& Miller, 2005; Ceballos et al., 2006; Kelly et al., 2008).

El resto de los felinos que se distribuyen en la Península han sido estudiados marginalmente; en el caso del puma, se ha determinado su distribución y nicho trófico solo en el sur de la Península (Chávez, Zarza, Ceballos, \& Amín, 2007). Por su parte, los trabajos sobre los felinos pequeños como el ocelote, tigrillo y jaguarundi, son descriptivos (Semarnat, 2002; Faller, 2007; Rodríguez-Reynaga et al., 2007; Sosa-Escalante, Pech-Canche, MacSwiney, \& Hernández-Betancourt, 2013).

Para fundamentar acciones de conservación de los felinos de la Península de Yucatán es imprescindible evaluar con más detalle sus poblaciones. Ya sea a través de medidas indirectas como la abundancia relativa (e.g., el número de rastros por unidad de esfuerzo) (Yasuda, 2004; Monroy-Vilchis, Zarco-González, Rodríguez-Soto, Soria-Díaz, \& Urios, 2011), o estimaciones de densidad, calculada por medio de técnicas de captura-recaptura (CR) que son muy utilizadas y analizadas con el programa CAPTURE (Otis, Bumham, White, \& Anderson, 1978; Rexstad \& Burnham, 1991), para datos provenientes del trampeo fotográfico (Karanth \& Nichols, 1998). Este método ha sido el más empleado para el estudio de felinos silvestres (Karanth \& Nichols, 1998; Kelly, 2003; Maffei, Cuellar, \& Noss, 2004; Silver et al., 2004; Chávez et al., 2007; Salom, Carrillo, Sáenz, \& Mora, 2007; Dillon \& Kelly, 2008; Noss et al., 2012).

Recientemente, se ha demostrado que estos procedimientos tienden a subestimar o sobrestimar los valores reales de la densidad (Tobler \& Powell, 2013). Por lo que se ha generado un modelo espacialmente explícito (SECR, por sus siglas en inglés) (Borchers \& Efford, 2008) implementado en el programa SPACECAP (Gopalaswamy et al., 2012). Su uso apenas se esta generalizando, por lo que es importante comparar su desempeño con modelos anteriormente empleados.

Los objetivos del presente estudio fueron: 1) estimar la densidad poblacional de los principales felinos silvestres del Norte de la Península de Yucatán, 2) comparar los resultados de densidad obtenidos por tres modelos, dos con base en el modelo de CR tradicionales (MMDM y CR-1/2MMDM) y otro en el de SECR, y con estudios previos, y 3) validar la importancia de esta región de México para la conservación de dichas especies.

\section{MATERIALES Y MÉTODOS}

Área de estudio: La Reserva Ecológica El Edén (REEE) abarca 3077 ha en el municipio de Lázaro Cárdenas, Quinta Roo, en el noreste

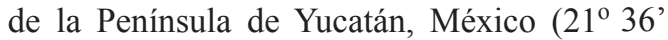
$00^{\prime \prime} \mathrm{N}-87^{\circ} 45^{\prime} 00^{\prime}$ W). La Reserva pertenece a la región biológica de Yalahau (Gómez-Pompa, Allen, Fedick, \& Jiménez-Osorio, 2003; 
Gómez-Pompa, Lazcano-Barrero, GómezBarrero, \& Macswiney, 2011), y tiene una altitud de 5-10 msnm (Lazcano-Barrero et al., 1992). Cuenta con cinco tipos de vegetación, de los que destacan por su extensión la selva mediana subcaducifolia y los acahuales. Esta zona forma parte de las selvas tropicales más norteñas del continente, una porción de las cuales se encuentra bajo resguardo federal dentro del Área de Protección de Flora y Fauna Yum Balam de 154052 ha (Navarro, Remolina, \& Pérez, 2007).

Diseño de muestreo y selección de sitios: El estudio se realizó de julio a septiembre 2008, octubre a diciembre 2010, mayo a julio 2011 y agosto a noviembre 2012. Se empleó el método de trampeo fotográfico. Las cámaras utilizadas fueron de las marcas Cuddeback Expert, Capture, Capture IR, Moultrie y Wildview. Se siguió el diseño del CENJAGUAR (Chávez et al., 2007); y se seleccionaron dos o tres estaciones (sitios donde se colocan las cámaras) en una parcela de $9 \mathrm{~km}^{2}$; se establecieron al menos nueve de estas, contiguas; y en cada una, por lo menos una estación fue "doble" (i.e., con dos cámaras, una frente a la otra). En el 2008, se dispusieron 27 estaciones que estuvieron activas 65 días; en 2010, 24 por 48 días; en 2011, 22 por 82 días y, finalmente, en el 2012, 24 que funcionaron durante 72 días. Las estaciones fueron ubicadas en senderos, brechas corta fuego y caminos de terracería, en donde se observaron rastros de felinos o sus presas potenciales, su colocación fue siguiendo la metodología de Silver (2004). Las estaciones fueron situadas a una distancia de 1.5 a $3 \mathrm{~km}$ una de otra. Las cámaras operaron las 24 horas del día y fueron programadas para sacar fotografías cada 30 segundos, se revisaron cada 15 días para asegurar su correcto funcionamiento.

Identificación de los individuos: Los felinos fotografiados fueron diferenciados entre sí, ya sea por su patrón de rosetas individuales en el caso de jaguares, ocelotes y tigrillos, o en el caso de los pumas por marcas conspicuas, como la forma de la cola, cicatrices, muescas en las orejas, manchas en la parte interna de las patas y la forma y porte del animal (Kelly et al., 2008).

Abundancia relativa: Se utilizó el número de registros independientes de cada una de las especies por unidad de esfuerzo para determinar un índice de abundancia relativa (AR - registros por 1000 trampas-día) de las cinco especies (Maffei, Cuellar, \& Noss, 2002; Yasuda, 2004; Monroy-Vilchis et al., 2011).

En todos los análisis se utilizaron registros (eventos) independientes de cada especie, tales como: 1) en las fotografías consecutivas que no fue posible identificar a cada animal de la misma especie como individuo distinto, se consideraron fotografías independientes a cada una de ellas con una separación de tres horas entre un evento y otro, 2) fotografías consecutivas de individuos diferentes de la misma especie plenamente identificables y 3) una fotografía con múltiples individuos, se consideró a cada uno de los ejemplares un evento.

Densidad: Para poder estimar la densidad de los felinos, primero se obtuvieron las abundancias generadas por el programa CAPTURE (Rexstad \& Burnham, 1991); este hace uso de la probabilidad de captura $(p)$ para producir estimaciones del tamaño de la población, bajo dos supuestos: 1) la población es cerrada demográfica y geográficamente y 2) todos los individuos tienen una probabilidad de captura mayor a cero, esto al considerar que se coloca una cámara por $10 \mathrm{~km}^{2}$, el área mínima de acción reportada para un jaguar (Silver, 2004). Para asegurar la primera condición, el periodo de estudio por año fue menor a 90 días. Además, CAPTURE realiza una serie de pruebas para evaluar la heterogeneidad en la probabilidad de captura que se puede dar por el comportamiento. En esta etapa, los datos se corren con cada modelo y se asigna un valor de 0 a 1 a cada uno, donde el valor más alto indica que el modelo tuvo un mejor comportamiento.

Los modelos que explican mejor el comportamiento de los individuos son el $\mathrm{Mh}$ (heterogeneidad individual), que adjudica 
probabilidades de captura diferentes para cada individuo, las cuales permanecen igual a través de cada ocasión de muestreo, y el M0 ("modelo nulo") que asume que no hay variación en la probabilidad de capturas asociadas a los individuos u ocasiones (Karanth \& Nichols, 1998, 2000, 2002); estos modelos usan el estimador jackknife y nulo, respectivamente (ver Otis et al., 1978); de estos dos, se eligió aquel que mejor se ajustó a nuestra información.

Posteriormente, la densidad de los felinos se estimó dividiendo la abundancia relativa generada por CAPTURE entre el AEM. Esta última se construyó adicionando un buffer (o zona de amortiguamiento) al polígono mínimo convexo (PMC) cubierto por las estaciones de fototrampeo. El área que cubrió el PMC fue de $63.0 \mathrm{~km}^{2}$ en $2008,47.8 \mathrm{~km}^{2}$ en $2010,54.0 \mathrm{~km}^{2}$ en 2011 y $75.1 \mathrm{~km}^{2}$ en 2012 .

Se estimó el AEM por medio de dos aproximaciones: 1) la media de la distancia máxima de movimiento de aquellos animales capturados en más de dos estaciones de fototrampeo (MMDM, por sus siglas en inglés) y 2) la mitad de esa medida ( $1 / 2 \mathrm{MMDM})$ como distancia buffer (Silver, 2004). La estimación del AEM se hizo con el software ArcView 3.2 (ESRI, 1994).

Adicionalmente, se estimó la densidad por medio de modelos espacialmente explícitos del paquete SPACECAP (Gopalaswamy et al., 2012). Este estima directamente la densidad utilizando tanto las historias de captura de los individuos, como los sitios específicos de las estaciones de trampeo. En SPACECAP se especificó el modelo nulo con una función de detección media normal y un proceso binomial de presencias. En todos los análisis se utilizaron las especificaciones propuestas por Noss et al. (2012) ya que no se contaba con suficientes datos para incluir covariantes y además se querían comparar los resultados utilizando los mismos modelos.

Se utilizaron los tres modelos para que los resultados pudieran ser comparados con trabajos realizados con anterioridad (en el caso de MMDM y $1 / 2$ MMDM) en otras áreas de estudio (Maffei et al., 2004; Noss, Kelly,
Camblos, \& Ruiz, 2004; Di bitetti, Paviolo, \& De Angelo, 2006; Chávez et al., 2007; Dillon \& Kelly, 2008; Kelly et al., 2008), y SERC (SPACECAP) como un modelo más robusto y menos sesgado (Noss et al., 2012; Tobler \& Powell, 2013; Tobler, Carrillo-Percastegui, Zúñiga, \& Powell, 2013).

\section{RESULTADOS}

El porcentaje de captura considerando todos los registros independientes de las cinco especies de felinos fueron: $48.4 \%, 23.1 \%$, $13.6 \%$ y $45.7 \%$ de jaguares; $26.2 \%, 29.2 \%$, $53.4 \%$ y $31.5 \%$ de pumas; $23.2 \%, 33.8 \%$, $30.8 \%$ y $18.9 \%$ de ocelotes y $2.0 \%, 12.3 \%$, $2.2 \%$ y $3.9 \%$ de tigrillos, respectivamente por año de estudio; solo se obtuvo una fotografía de jaguarundi, que correspondió al $1.5 \%$ de los registros de felinos en 2010. En el caso de los tres felinos mayores, hubo variaciones en el número de estaciones donde se fotografiaron individuos por especie. Para jaguares, en 2008 y 2012 se registró en un mayor número de estaciones a diferencia de los otros dos años, cuyos registros fueron en menos de la mitad de las estaciones. Para los pumas, solamente en el 2010 se ve esta reducción en el número de estaciones donde fueron registrados; y en el caso de ocelotes, los registros variaron entre 12 y 13 estaciones (Cuadro 1, Fig. 1, Fig. 2, Fig. 3). En total, durante los cuatro años se registraron 24 jaguares, 40 pumas y 22 ocelotes. El número de individuos identificados y recapturados varió a lo largo de los cuatro años (Cuadro 1).

La AR para las cinco especies, durante los cuatro años, fue muy variable, con cifras mayores en 2008 para las tres especies: jaguares 44.9 (registros/1 000 trampas-días); pumas 24.3; ocelotes 21.5; y en el 2010 para las otras dos: tigrillos 3.7 y jaguarundi 0.4 (Fig. 4).

Para los jaguares y los ocelotes, las abundancias estimadas por CAPTURE más altas fueron en 2010, $5.0 \pm 1.4,19 \pm 21.9$, mientras que para pumas la más alta fue en el 2008, $8.5 \pm 4.2$. A su vez, las abundancias más altas coinciden con los intervalos de confianza más amplios (Cuadro 2). 

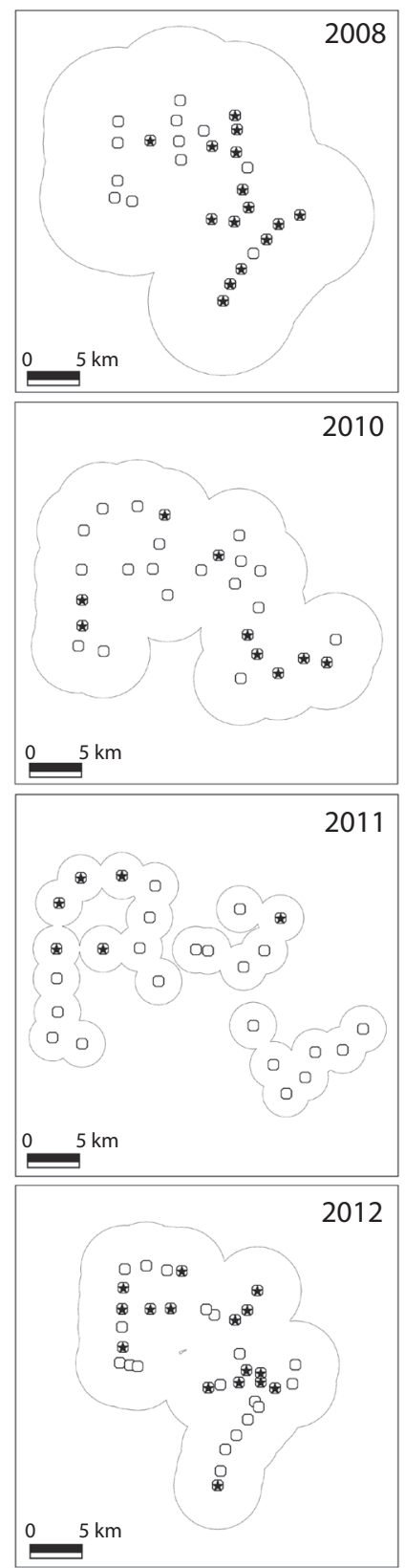

$\star$ Jaguares

○ Cámaras

Fig. 1. Estaciones de trampeo por año de muestreo, área efectiva de muestreo usando MMDM para jaguares y las estaciones donde fue capturada la especie en la Reserva Ecológica el Éden, Quintana Roo, México.

Fig. 1. Camera stations by sampling years; effective sampling area using MMDM of jaguar and stations where the specie was captured in El Eden Ecological Reserve, Quintana Roo, Mexico.
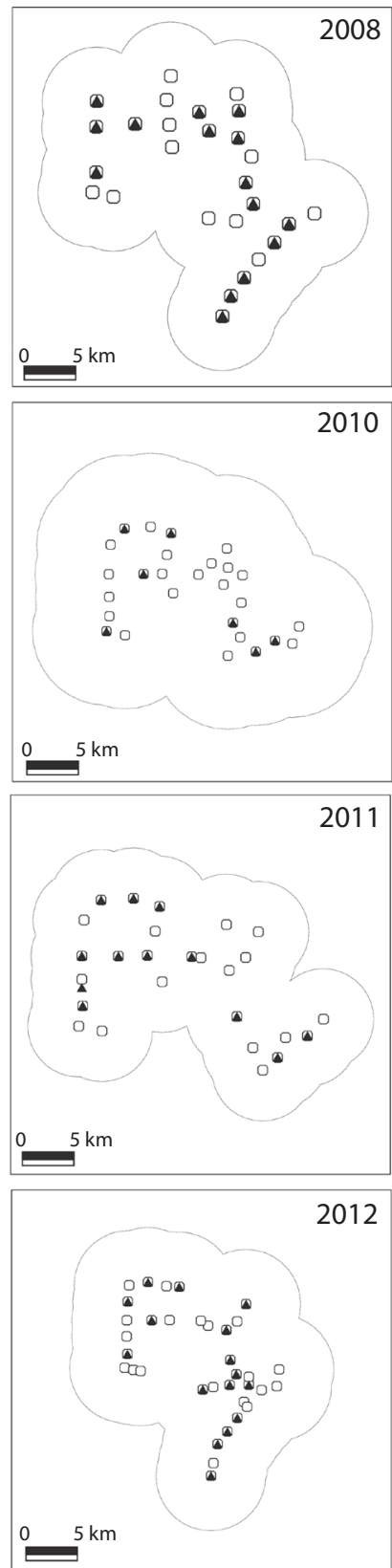

- Puma o Cámaras

Fig. 2. Estaciones de trampeo por año de muestreo, área efectiva de muestreo usando MMDM para pumas y las estaciones donde fue capturada la especie en la Reserva Ecológica el Éden, Quintana Roo, México

Fig. 2. Camera stations by sampling years, effective sampling area using MMDM of pumas and stations where the specie was captured in El Eden Ecological Reserve, Quintana Roo, Mexico. 

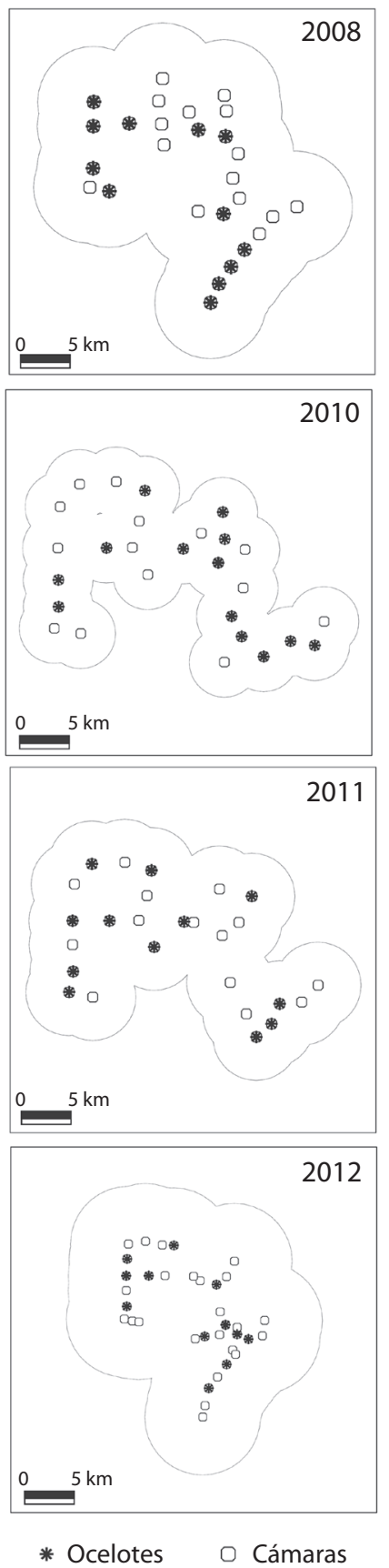

Fig. 3. Estaciones por año de muestreo, área efectiva de muestreo usando MMDM para ocelotes y las estaciones donde fue capturada la especie, en la Reserva Ecológica el Éden, Quintana Roo, México.

Fig. 3. Locations of the camera stations by sampling years; effective sampling areas using MMDM of ocelots and stations where the specie was captured in El Eden Ecological Reserve, Quintana Roo, Mexico.
Densidad: La densidad se calculó solo para jaguares, pumas y ocelotes debido al número de fotografías independientes, a los requisitos del programa y de los modelos empleados. Al comparar los valores obtenidos por los tres modelos (MMDM, 1/2MMDM y SECR) para las tres especies, se encontró que las estimaciones realizadas con $1 / 2$ MMDM y MMDM, son mayores que las generadas por SECR-SPACECAP, excepto para pumas en el 2012. Al contrastar los resultados de MMDM con los de SPACECAP, se observa que el $50 \%$ de las densidades generadas por la primera fueron menores a las calculadas por SPACECAP - jaguares 2011 y 2012, pumas 2010 a 2012 y ocelotes en 2012 (Cuadro 3).

En general, las densidades más altas para los jaguares fueron encontradas en el 2010, calculadas por $1 / 2$ MMDM (5.5 ind./100 $\mathrm{km}^{2}$ ) y MMDM (2.6 ind./100 km²) y el 2011 para SECR-SPACECAP (3.5 ind./100 km²). En 2008, se calcularon las densidades menores por los tres modelos y fluctuaron entre 0.7 a 2.6 ind./100 (Cuadro 3). Por su parte, la densidad de pumas varió entre 1.7 ind. $/ 100 \mathrm{~km}^{2}$ con MMDM en el 2010 a 8.5 ind. $/ 100 \mathrm{~km}^{2}$ con $1 / 2$ MMDM en el 2008. La densidad de ocelotes fue más variable que las de las otras especies, ya que osciló entre 1.4 (2012) y 13.8 ind./100 $\mathrm{km}^{2}$ (2010) (Cuadro 3).

En contraste, las estimaciones realizadas por SPACECAP respecto a las de MMDM muestran coincidencias en las realizadas para jaguares en 2010, y ocelotes 2012, las cuales son las más bajas, a diferencia con las estimadas para pumas en 2008 (Cuadro 3).

\section{DISCUSIÓN}

Con este estudio se obtuvieron por primera vez estimaciones de AR de las cinco especies de felinos reportadas para el norte de la Península de Yucatán, y de densidad para tres de ellas. A lo largo de los cuatro años de estudio, cuando el AR del ocelote es mayor, el del tigrillo decrece, y cuando se mantienen constantes las AR de ambas especies, la del tigrillo se mantiene por debajo de la del ocelote. En el 


\section{CUADRO 1}

Número de individuos de jaguares (Panthera onca), pumas (Puma concolor) y ocelotes (Leopardus pardalis) por año, en la Reserva Ecológica El Edén, Quintana Roo, México

TABLE 1

Number of individuals of jaguar (Panthera onca), puma (Puma concolor), and ocelot (Leopardus pardalis) per year, in El Edén Ecological Reserve, Quintana Roo, Mexico

Individuos (M:H:N)*

Total de individuos

Capturas

Total de capturas

Número de estaciones con capturas

Individuos (M:H:N)

Total de individuos

Capturas

Total de capturas

Número de estaciones con capturas

Individuos (M:H:N)

Total de individuos

Capturas

Total de capturas

Número de estaciones con capturas

Individuos (M:H:N)

Total de individuos

Capturas

Total de capturas

Número de estaciones con capturas

\begin{tabular}{cccc} 
Jaguar & & Puma & Ocelote \\
& $\mathbf{2 0 0 8}$ & & \\
$4: 2: 1$ & & $5: 3: 5$ & $6: 3: 1$ \\
7 & 13 & 10 \\
$55: 40: 1$ & $38: 6: 11$ & $24: 16: 1$ \\
96 & 55 & 41 \\
16 & & 15 & 12 \\
& $\mathbf{2 0 1 0}$ & & \\
$3: 1: 1$ & & $3: 2: 3$ & $4: 4: 2$ \\
5 & & 7 & 10 \\
$7: 6: 1$ & & 17 & $9: 7: 2$ \\
14 & & 7 & 18 \\
9 & $\mathbf{2 0 1 1}$ & $4: 3: 4$ & 13 \\
$5: 0: 0$ & & 11 & $6: 4: 0$ \\
5 & & $29: 7: 24$ & 10 \\
$12: 0: 0$ & & 60 & $15: 11: 0$ \\
12 & & 12 & 26 \\
6 & $\mathbf{2 0 1 2}$ & $5: 4: 3$ & 12 \\
$6: 2: 4$ & & 12 & $2: 5: 2$ \\
12 & & $37: 9: 4$ & 9 \\
$51: 5: 6$ & 16 & $10: 18: 2$ \\
62 & & 30 \\
16 & & & 12 \\
\hline
\end{tabular}

* $\mathrm{M}=$ machos, $\mathrm{H}=$ hembras, $\mathrm{N}=$ no determinado $* \mathrm{M}=$ male, $\mathrm{F}=$ female, $\mathrm{N}=$ not determined.

\section{CUADRO 2}

Abundancia de jaguares (Panthera onca), pumas (Puma concolor) y ocelotes (Leopardus pardalis) por medio del programa CAPTURE, en la Reserva Ecológica el Edén, Quintana Roo, México

TABLE 2

Abundance of jaguars (Panthera onca), pumas (Puma concolor), and ocelots (Leopardus pardalis) according the program CAPTURE. El Eden Ecological Reserve, Quintana Roo, Mexico

\begin{tabular}{ccccc} 
Año & Abundancia $\pm S E$ & Intervalo de confianza 95 \% & Z & P \\
2008 & $6 \pm 0.43$ & Jaguares & -2.46 & 0.00 \\
2010 & $5 \pm 1.40$ & $6-6$ & 1.77 & 0.96 \\
2011 & $2 \pm 0.66$ & $5-13$ & -1.64 & 0.05 \\
2012 & $6 \pm 2.27$ & $2-6$ & -7.42 & 0.22 \\
& & & \\
2008 & $12 \pm 4.22$ & Pumas & -2.19 & 0.01 \\
2010 & $8 \pm 3.57$ & $9-32$ & 0.84 & 0.80 \\
2011 & $8 \pm 3.01$ & $7-26$ & 0.19 & 0.57 \\
2012 & $11 \pm 2.99$ & $8-25$ & -1.03 & 0.15 \\
2008 & $7 \pm 5.78$ & $11-29$ & & 0.01 \\
2010 & $19 \pm 21.9$ & Ocelotes & -2.24 & 0.77 \\
2011 & $12 \pm 2.63$ & $6-42$ & 0.74 & 0.29 \\
2012 & $8 \pm 1.11$ & $9-140$ & -0.52 & 0.47 \\
\hline
\end{tabular}




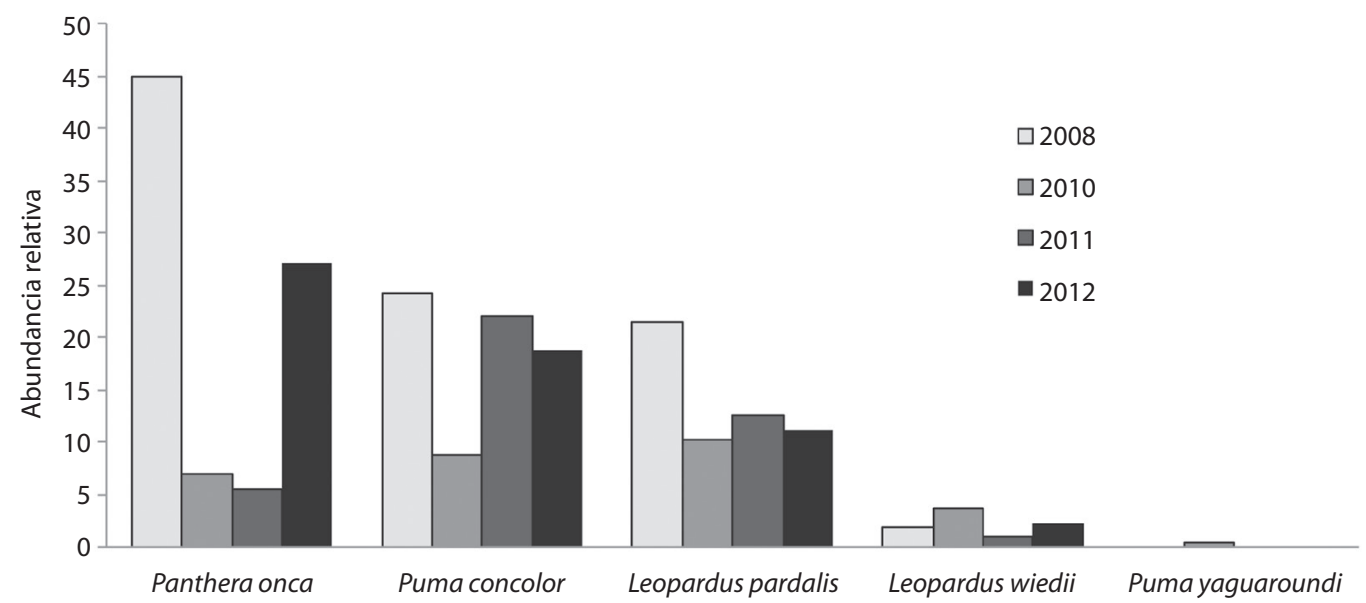

Fig. 4. Abundancia relativa (fotografías independientes/1000 trampas-día) de las cinco especies de felinos de la Reserva Ecológica El Eden, Quintana Roo, México.

Fig. 4. Relative abundance (independent photographs/1000 trap-day) of the five felids in El Eden Ecological Reserve, Quintana Roo, Mexico.

\section{CUADRO 3}

Densidades de jaguares (Panthera onca), pumas (Puma concolor) y ocelotes (Leopardus pardalis) obtenidas por medio de CAPTURE + MMDM y 1/2 MMDM y SPACECAP en la Reserva Ecológica El Edén, Quintana Roo, México

TABLE 3

Densities of jaguars (Panthera onca), pumas (Puma concolor), and ocelots (Leopardus pardalis) obtained through CAPTURE + MMDM and 1/2MMDM and SPACECAP for four years of study in the El Eden Ecological Reserve, Quintana Roo, Mexico

\begin{tabular}{|c|c|c|c|c|c|c|c|c|}
\hline \multirow{3}{*}{ Año } & \multicolumn{6}{|c|}{ CAPTURE } & \multicolumn{2}{|c|}{ SPACECAP } \\
\hline & \multicolumn{3}{|c|}{ AEM con MMDM } & \multicolumn{3}{|c|}{ AEM con $1 / 2$ MMDM } & \multirow{3}{*}{$\mathrm{D} \pm \mathrm{SE}$} & \multirow{3}{*}{$\lambda 0$} \\
\hline & $\operatorname{MMDM}(\mathrm{km})$ & $\mathrm{AEM} \mathrm{km}{ }^{2}$ & $\mathrm{D}+\mathrm{SE}$ & $1 / 2 \operatorname{MMDM}(\mathrm{km})$ & $\mathrm{AEM} \mathrm{km}{ }^{2}$ & $\mathrm{D}+\mathrm{SE}$ & & \\
\hline \multicolumn{7}{|c|}{ Jaguares } & & \\
\hline 2008 & 7.8 & 496 & $1.20 \pm 0.43$ & 3.93 & 226 & $2.65 \pm 0.43$ & $0.70 \pm 0.05$ & 0.00890 \\
\hline 2010 & 3.7 & 190 & $2.63 \pm 1.40$ & 1.85 & 99 & $5.05 \pm 1.40$ & $1.10 \pm 0.71$ & 0.0009 \\
\hline 2011 & 1.5 & 88 & $2.27 \pm 0.66$ & 0.79 & 42 & $4.76 \pm 0.66$ & $3.65 \pm 1.39$ & 0.0036 \\
\hline 2012 & 4.9 & 315 & $1.58 \pm 2.27$ & 2.48 & 162 & $3.08 \pm 2.27$ & $2.33 \pm 0.88$ & 0.0059 \\
\hline \multicolumn{9}{|c|}{ Pumas } \\
\hline 2008 & 4.8 & 277 & $4.33 \pm 4.22$ & 2.41 & 140 & $8.57 \pm 4.22$ & $1.85 \pm 0.53$ & 0.0039 \\
\hline 2010 & 7.8 & 453 & $1.76 \pm 3.57$ & 3.90 & 200 & $4 \pm 3.57$ & $2.90 \pm 1.26$ & 0.0089 \\
\hline 2011 & 6.5 & 374 & $2.13 \pm 3.01$ & 2.18 & 118 & $6.77 \pm 3.01$ & $3.67 \pm 1.34$ & 0.0029 \\
\hline 2012 & 7.0 & 467 & $2.35 \pm 2.99$ & 3.51 & 222 & $4.95 \pm 2.99$ & $5.17 \pm 1.48$ & 0.0019 \\
\hline \multicolumn{9}{|c|}{ Ocelotes } \\
\hline 2008 & 5.3 & 310 & $2.25 \pm 5.70$ & 2.66 & 153 & $4.57 \pm 5.7$ & $2.10 \pm 0.66$ & 0.0010 \\
\hline 2010 & 2.6 & 137 & $13.86 \pm 21.90$ & 1.32 & 73 & $26.02 \pm 21.9$ & $4.40 \pm 1.56$ & 0.0090 \\
\hline 2011 & 3.6 & 195 & $6.15 \pm 2.60$ & 1.83 & 101 & $11.88 \pm 2.6$ & $5.15 \pm 1.55$ & 0.0050 \\
\hline 2012 & 8.1 & 561 & $1.42 \pm 1.10$ & 4.07 & 277 & $2.88 \pm 1.1$ & 1.66 & - \\
\hline
\end{tabular}

Buffer = distancia agregada al polígono de cámaras para generar el área efectiva de muestreo; $\mathrm{D}=$ número de individuos $/ 100$ $\mathrm{km}^{2} ; \lambda 0=$ tasa de captura esperada. Se convirtio $\lambda$ a una probabilidad de captura usando 1 -exp $(-\lambda 0)$, la cual es practicamente igual cuando $\lambda 0$ es un valor muy bajo.

Buffer $=$ distance added to camera polygon to generate effective sampling area; $\mathrm{D}=$ number of individuals $/ 100 \mathrm{~km}^{2} ; \mathrm{Lam} 0$ $=$ expected capture rate. $\lambda$ was converted a capture probability using $1-\exp (-\lambda 0)$, which is almost the same when $\lambda 0$ is a very low value. 
caso de los grandes felinos, la AR del jaguar es superior a la del puma en dos años, pero cuando el AR del jaguar decrece la del puma aumenta. Los registros de jaguaroundi solamente se limitan al 2010 y su AR es la más baja, tal como ha pasado en otros estudios (Maffei et al., 2004).

De manera general, el modelo SECR generó los valores de densidades más conservadores, por ejemplo, la densidad de jaguares varió de 0.3-3.6 ind. $/ 100 \mathrm{~km}^{2}$, la de pumas de $1.8-5$ ind. $/ 100 \mathrm{~km}^{2}$ y la de ocelotes entre 1.6-5.1 ind. $/ 100 \mathrm{~km}^{2}$; todos estos valores se encuentran dentro de los intervalos reportados en la literatura (Noss et al., 2004; Faller, Chávez, Johnson, \& Ceballos, 2007; Kelly et al., 2008). Sin embargo, la mayoría de los estudios publicados reportan densidades evaluadas en periodos de uno o dos años, mientras que los obtenidos en este trabajo fueron a mediano plazo y en la misma área. La variabilidad de las densidades, no obstante al haber utilizado modelos estandarizados, indican la necesidad de que los estudios sobre depredadores ápice se realicen a largo plazo. Solo así se podrá comprender la variación en la presencia y proporción de los distintos felinos, las cuales pueden depender de la temporada y año de la estimación, cambios ambientales, conductuales, interacciones entre las distintas especies de felinos y sus presas y efectos humanos.

Los modelos de CR que utilizan el AEM ( $1 / 2$ MMDM y MMDM) tienden a generar una valoración poco precisa de la densidad debido a que utilizan poca información para calcular el buffer, sobre todo cuando se trabaja en un área (grupo de estaciones) menor al área de actividad de la especie, estos métodos tienden a sobreestimar la densidad (Sollman et al., 2012; Tobler \& Powell, 2013). Además, los modelos convencionales de marcaje-CR suelen presentar una dependencia del tamaño de muestra (individuos capturados y sus recapturas en múltiples estaciones) (Gopalaswamy et al., 2012). Al contrastar los modelos de CR tradicionales con los modelos de captura recaptura espacialmente explícitos (SECR) se hace patente que los modelos SECR producen estimaciones más conservadoras (Tobler et al., 2013) mientras que en los modelos tradicionales, se pierde la información espacial derivada de la localización de las estaciones de captura, y la falta de información crítica relacionada al área de actividad o de los movimientos espaciales de los animales (Karanth \& Nichols, 1988, Gopalaswamy et al., 2012). Otra de las ventajas de usar SECR es que es más robusto al tener pocos individuos y pocas recapturas que los modelos tradicionales de CR (Gopalaswamy et al., 2012) y es menos sensible al tamaño pequeño de las cuadrículas (Tobler et al., 2013).

En el caso de la REEE, el intervalo de densidades calculado para jaguares fue de 2.6 a 5.0 ind. / $100 \mathrm{~km}^{2}$ por $1 / 2$ MMDM, valores muy parecidos a los reportados en otros sitios de la región, como los de la Reserva de la Biosfera Ría Lagartos (RBRL), el Zapotal, Yum Balam y Dzilam (Faller et al., 2007); zonas con las que mantiene conectividad y con las cuales pueden estar compartiendo el ámbito hogareño de los individuos. De igual manera, son similares a los del sur de la Península de Yucatán (Ceballos, Chávez, Rivera, \& Manterola, 2002; Chávez et al., 2007).

El intervalo de la densidad calculada (0.7 a 5.0 ind. $/ 100 \mathrm{~km}^{2}$ ) con los tres modelos (MMDM, 1/2 MMDM o SECR) en la REEE, queda entre las estimaciones realizadas en Bolivia y Belice (Noss et al., 2004), en los sitios que se utilizó un área de muestreo relativamente similar y comparables con áreas en otros estudios.

Los resultados obtenidos por SPACECAP variaron a lo largo de los cuatro años de estudio, oscilando entre 0.3 y 3.6 ind. / $100 \mathrm{~km}^{2}$. Dichas cifras son mayores a las obtenidas para el bosque seco, Bolivia (Noss et al., 2012). En contraste, la densidad más alta en la REEE, 3.6 ind. $/ 100 \mathrm{~km}^{2}$, se encuentra por debajo de la reportada para Perú y Brasil (Tobler \& Powell, 2013; Tobler et al., 2013).

La variación en la densidad durante los cuatro años consecutivos es amplia, similar a lo que ocurre en otros estudios; por ejemplo, en Los Amigos, Perú, cuya densidad varia de 4.0 a 9.0 (SERC) (Tobler et al., 2013). Los autores argumentan que una baja tasa de encuentros 
junto con un tamaño pequeño de cuadrícula puede resultar en una estimación de densidad altamente sesgada (Tobler et al., 2013). Sin embargo, la comparación que ellos hacen de densidad a través de los años con una cuadrícula del mismo tamaño, provocó una estimación diferente. Por lo tanto, no solo el cálculo de densidad depende de estos dos factores, sino de la dinámica propia de las poblaciones y del ambiente, es decir, la época del año y los efectos ambientales; en la REEE estos dos factores influyeron en los resultados ya que los jaguares mostraron en el 2010 y 2011 un tasa de captura baja ocasionada por incendios cerca de la reserva, que provocan una movilidad de la especie y de sus presas a zonas al norte de la Reserva. A diferencia de los otros dos años donde la tasa de encuentros fue alta, pero aun así, en el 2008 se capturaron cinco individuos y en el 2012, 12.

En muestreos realizados en diferentes años en la misma temporada, se han dado variaciones muy grandes en la estimación de la abundancia de 3.3 a 12.2 en los Amigos, Perú (Tobler et al., 2013); estas variaciones son en parte determinadas por el número de estaciones muestreadas, siendo las estimaciones más altas ocasionadas por un área de muestreo relativamente pequeña, en comparación con la de los dos años subsiguientes, en los cuales la estimación es relativamente parecida (3.3 y 3.9). Nuestros datos por una parte muestran un patrón un poco diferente, aún cuando tenemos años (2008 y 2010) con meses muy parecidos, las densidades son muy distintas entre $0.7 \mathrm{y}$ 2.3 , esto probablemente nos esté indicando que aunque los muestreos sean en la misma temporada, pueden existir cambios anuales en la presencia de individuos en el área, ya que tenemos el mismo número de estaciones con capturas en esos años, pero lo que cambia es en el número de individuos que está presente en el área.

Los estudios para estimar la densidad de pumas no han sido comunes en México. En general, las densidades varían por área y, en algunos casos, como el norte de la Península, presentan densidades dentro de los rangos encontrados en otras zonas de Latinoamérica. Por ejemplo, en la REEE varió de 4.0 a 8.5 ind./100 $\mathrm{km}^{2}$ (1/2MMDM, Mh), valores que estan por arriba de los obtenidos por Noss et al. (2012) o intermedios como en ciertas zonas de Belice y Bolivia (Noss et al., 2004). Por otra parte, Kelly et al. (2008) reportan densidades que varían entre países, por ejemplo las de Argentina y Belice fueron inferiores a las reportadas en este trabajo, mientras que las de Bolivia fueron superiores.

Los estudios que reportan densidades de pumas calculadas por SECR (SPACECAP) son aún muy pocos. La densidad obtenida en la REEE van de 1.8 a 5.1 ind. $/ 100 \mathrm{~km}^{2}$ que son densidades superiores a las reportadas por Noss et al. (2012) para el Gran Chaco, Bolivia.

La densidad de ocelotes en la REEE varió también considerablemente durante el estudio; osciló entre 1.4 y 13.8 ind./100 km² (MMDM). En la misma reserva se han calculado densidades de 12.7 ind./100 km² (Torres-Romero, 2009). El valor más bajo fue inferior a los reportados en otras partes de su distribución; por ejemplo, en Urugua-í e Iguazú, Argentina, (Di Bitetti et al., 2006). Sin embargo, en el 2010 se presenta la mayor densidad, similar a la máxima reportada por Di Bitetti y colaboradores en 2006. Por otro lado, en la Estación de Investigación Las Cuevas, Belice, Dillon y Kelly (2008) reportaron una densidad similar a la máxima registrada en El Edén. En Los Llanos de Colombia, se encontró una densidad de 5.4 ind./100 $\mathrm{km}^{2}$ (MMDM); los autores argumentan que es una de las densidades más bajas para la especie debido al tipo de hábitat: bosque de galería, sabanas abiertas y tierras de uso agrícola (Díaz-Pulido \& Payán, 2011). Sin embargo, en el presente estudio se reportan densidades aún más bajas para el 2008 y 2012, esto posiblemente debido a la dinámica poblacional y a la interacción con los co-depredadores.

En contraste, las densidades por medio de SECR tuvieron un rango de 1.6 a 5.1 ind. / $100 \mathrm{~km}^{2}$ que son inferiores a los obtenidos por MMDM y 1/2 MMDM, con excepción del 2012 que está ligeramente por debajo al obtenido por $1 / 2$ MMDM. Las densidades de SECR en 
la REEE son similares a las del Chaco, Bolivia (Noss et al., 2012).

En general, los estudios comparativos demuestran que las densidades de felinos varían entre especies, lugares o tipos de vegetación. Por ejemplo, un contraste entre pumas y jaguares (Noss et al., 2004) en bosques secos (Bolivia) y bosques húmedos (Belice) señala que los primeros tienen una mayor densidad (2.9-10.5 ind./100 $\mathrm{km}^{2}$ ) que los segundos (0.214.6), mientras que en Belice los jaguares tienden a ser más numerosos (2.3-7.4) que los pumas (2.8-4.3). Para el Norte de México, Rosas-Rosas y Bender (2012) encontraron que los pumas (1.7) son más abundantes que los jaguares (1.1).

El jaguarundi fue escaso, ya sea porque no existe el hábitat adecuado o porque el método o diseño de muestreo no fue efectivo. A pesar del esfuerzo de muestreo realizado, solo se pudo obtener un registro de esta especie, en el 2010; en otros estudios también ha sido fotografiado marginalmente (Maffei et al., 2004). El jaguarundi es considerado como una especie rara y de comportamiento diferente al de otros felinos (Maffei, Noss, \& Fiorello, 2007). Por lo tanto, la falta de registros podría no reflejar su abundancia sino más bien sus hábitos elusivos. En Bolivia, se reporta como una especie común pero difícil de ver (Tello, 1986); en México se le describe como una especie adaptable y resistente a la fragmentación y destrucción del hábitat (Aranda, 1991) y, al parecer, se encuentra más asociada a ecotonos entre bosque y zonas abiertas (Aranda, 2005a).

El tigrillo fue registrado durante los cuatro años de estudio, pero su AR fue baja y, por lo tanto, presentó una tendencia opuesta a la del ocelote, al menos durante los tres primeros años. En general, esta especie ha sido muy poco estudiada y se le caracteriza más como arborícola que terrestre, lo cual dificulta su estudio, particularmente con cámaras trampa. La mayoría de los estudios solo reportan su presencia en áreas tropicales y zonas de vegetación densa (Aranda, 2005b).

Para la conservación de los felinos, se debe tomar en cuenta los efectos que tienen las alteraciones naturales y humanas, en la presencia, movimientos y densidad de las especies. En la Península de Yucatán existen periódicamente alteraciones naturales que modifican el paisaje en diferentes grados; tres de los que ocurren con mayor frecuencia en el Norte de la Península de Yucatán son los huracanes, las inundaciones estacionales y los incendios forestales. Este último es una consecuencia de factores como sequías extremas, prácticas agrícolas de roza-tumba y quema y acumulación de materia vegetal, entre otras (Sánchez \& Rebollar, 1999). Entre los efectos que causan los incendios forestales a la fauna silvestre están la movilidad y dispersión de las especies (Faller et al., 2007). Por ejemplo, en las afueras del Edén se presentó un incendio durante mayo 2011; el incendio abarcó más de mil hectáreas al sur de la colindancia con la reserva afectando acahuales, selvas inundables y sabanas. Esta alteración tuvo un alto impacto en la movilidad de los animales $\mathrm{y}$, por ende, en las tasas de captura.

Se ha sugerido que la magnitud del fuego en los diferentes hábitats afecta la presencia y densidad de los felinos, pero el mayor efecto es sobre el jaguar (Michalski \& Peres, 2005), nosotros no podemos determinar esto con nuestros datos, ya que si bajó la tasa de captura e individuos, esta es muy parecida a la del 2010. Lo que encontramos es que, luego del incendio en la reserva en el 2011, los jaguares solo se registraron en la porción norte del área protegida y la tasa de captura fue la más baja. Los pumas también se vieron afectados en sus movimientos, sin embargo, se fotografiaron en el centro y norte de la reserva. Los ocelotes, en cambio, fueron los menos afectados ya que se les capturó en todas las zonas de la REEE; la tasa de captura de L. pardalis se mantuvo constante entre 2010-2011. Además, se debe considerar que los felinos responden a la abundancia y diversidad de presas, las cuales se ven también afectadas por los incendios (Fisher \& Wilkinson, 2005).

En la REEE se cuenta con cinco de los seis felinos reportados en México, las densidades obtenidas para jaguares, pumas y ocelotes son 
comparables a las registradas en otras partes de la Península y otros sitios como Belice y Guatemala. La REEE se ubica en una región importante que permite se mantenga la conectividad entre el norte y el este de la Península de Yucatán, por lo que juega un papel muy importante en el mantenimiento de la viabilidad de las poblaciones de esta región, en un paisaje cada vez más fragmentado.

Los estudios a largo plazo nos permiten conocer la dinámica de las poblaciones en una misma área, en donde la densidad se ve afectada en primer lugar por la dinámica de las especies dentro de determinada área, por la temporada de muestreo, las interacciones con codepredadores y presas, alteraciones en el ambiente ya sea naturales o humanas.

Por otra parte, la abundancia relativa de los felinos dentro de la REEE entendida como registros independientes entre esfuerzo de muestreo no refleja la densidad calculada por los tres métodos utilizados. El SECR/ SPACECAP hace una estimación de la densidad por debajo o igual a la del MMDM. La época lluviosa o seca incide directamente en la movilidad de los individuos, lo que afecta la distancia máxima de movimiento y, con ello, en las estimaciones realizadas por medio del MMDM y $1 / 2$ MMDM. Asimismo, influye en la dinámica poblacional y en el número de individuos que son fotografiados. Una baja tasa de recapturas influye directamente en el cálculo de la densidad pese al número de individuos identificados, por ende, puede resultar en una sobreestimación de este parámetro, en el caso de CAPTURE.

Finalmente, la dinámica temporal de las poblaciones de felinos en la REEE y su alrededores, es posible que existan zonas aledañas que, junto con la reserva, son importantes reservorios de poblaciones de felinos, por lo que, sin duda, el área es de gran importancia. Además, la conservación de dicha fauna dentro de la REEE y sus alrededores se garantizará si se mantienen las selvas medianas subcaducifolias, a su vez de permitir la recuperación de algunas áreas naturales perturbadas, lo que a su vez, le dará continuidad con otras reservas o zonas conservadas aun no alteradas, las cuales en conjunto mantienen importantes poblaciones de felinos y sus presas.

\section{AGRADECIMIENTOS}

A la Reserva Ecológica el Edén, el Centro de Investigaciones del Trópico de la Universidad Veracruzana, Kathy Cabrero, Erik J. Torres, Juan Castillo, Alejandro Pacheco, Brady Hollinsgworth, al personal de la reserva, a los voluntarios de Global Vision Internacional. A CONACyT por la beca número 211454 y al Financiamiento del Proyecto Promep 54310009 a C. Chávez (UAM-PTC-333) y al Colegio de Postgraduados, a Germán Mendoza y José Luis Figueroa Velasco por la revisión y aportes hechos al trabajo.

\section{RESUMEN}

La estimación de la densidad de fauna silvestre permite tener una idea del estado de salud de las poblaciones y en algunos casos indica el estado de conservación de los ecosistemas. Los métodos de evaluación deben hacer estimaciones no sesgadas, ya que servirán de base para estrategias de conservación de especies clave. Algunas regiones en México han sido identificadas como áreas de alta prioridad para la conservación de especies con cierto nivel de riesgo, como es la Península de Yucatán (PY), donde prevalece la mayor población de jaguares en México. Sin embargo, poco se sabe acerca de la situación actual de los felinos amenazados y en peligro de extinción, como un grupo de especies que conviven en la parte noreste de la Península. Nuestro objetivo fue estimar la densidad de las poblaciones de felinos silvestres a mediano plazo en la Reserva Ecológica El Edén (EEER) y sus alrededores. Se llevaron a cabo muestreos con cámaras-trampa durante cuatro años (2008, 2010, 2011 y 2012), se usaron modelos de captura-recaptura para poblaciones cerradas (CAPTURA + MMDM o $1 / 2$ MMDM) y un modelo de C-R espacialmente explícito (CERC) por medio del paquete SPCACAP para realizar las estimaciones de densidad. Las especies estudiadas fueron: jaguar (Panthera onca), puma (Puma concolor), ocelote (Leopardus pardalis), jaguarundi (Puma yaguaroundi) y tigrillo (Leopardus wiedii). La frecuencia de captura se obtuvo para las cinco especies y la densidad para tres (individuos $/ 100 \mathrm{~km}^{2}$ ). La densidad estimada por medio de MMDM varió entre 1.2 y 2.6 para jaguares, pumas (1.7-4.3) y ocelotes (1.4-13.8). Las estimaciones de la densidad en SPACECAP variaron desde 0.7 hasta 3.6 para jaguares, de 1.8 a 5.2 para pumas y de 2.1 a 5.1 en ocelotes. El método de C-R espacialmente explícito (SECR, 
SPACECAP) tiene menos probabilidades de sobrestimar la densidad, lo que provee una herramienta útil en el proceso de planificación y toma de decisiones para la conservación de estas especies. La parte noreste de la Península de Yucatán mantiene poblaciones altas de felinos, la REEE y sus alrededores son clave para la conservación de este grupo de depredadores.

Palabras clave: especie clave, áreas prioritarias de conservación, densidad, abundancia, Leopardus, Panthera, Puma.

\section{REFERENCIAS}

Amit, R., Gordillo-Chávez, E., \& Bone, R. (2013). Jaguar and puma attack on livestock in Costa Rica. HumanWildlife Interactions, 7(1), 77-84.

Aranda, M. (1991). Felinos de México: estado actual de conocimiento y conservación. En Memorias del simposio Felinos de Venezuela. Biología, ecología y conservación. FUNDECI, Caracas, Venezuela.

Aranda, M. (2005a). Leoncillo. In G. Ceballos \& G. Oliva (Ed.), Los mamíferos silvestres de México (pp. 358359). México: FCE-CONABIO.

Aranda, M. (2005b). Margay. In G. Ceballos \& G. Oliva (Ed.), Los mamiferos silvestres de México (pp. 361362). México: FCE-CONABIO.

Beschta, R., \& Ripple, W. (2009). Large predators and trophic cascades in terrestrial ecosystems of the western United States. USA: Biological Conservation.

Borchers, D. L., \& Efford, M. (2008). Spatially explicit maximum likelihood methods for capture-recapture studies. Biometrics, 64,377-385.

Ceballos, G., Chávez, C., Rivera, A., \& Manterola, C. (2002). Tamaño poblacional y conservación del jaguar en la Reserva de la Biosfera de Calakmul, Campeche, México. In R. Medellin, A. Rabinowitz, C. Chetkiewicz, K. Redford, J. Robinson, E. Sanderson, \& A. Taber (Eds.), El jaguar en el Nuevo milenio (pp. 403-418). México: Fondo de Cultura Económica, Universidad Nacional Autónoma de México, Wildlife Conservation Society.

Ceballos, G., Chávez, C., Zarza, H., \& Manterola, C. (2005). Ecología y conservación del jaguar en la región de Calakmul. CONABIO. Biodiversitas, 62, 1-7.

Ceballos, G., Chávez, C., \& Zarza, H. (2011). El Jaguar en México. México D. F.: Alianza WWF/Telcel Conanp, UNAM, Servicios Ecológicos y Científicos.

Ceballos, G., Chávez, C., List, R., Medellín, R., Manterola, C., ... Alcántara, S. M. B. (2006). Proyecto para la conservación y Manejo del Jaguar en México, Serie: Proyectos de Recuperación de Especies Prioritarias.
No. 14. México: Dirección General de Vida Silvestre, Semarnat.

Chávez, C., Zarza, H., Ceballos, G., \& Amín, M. (2007). Ecología poblacional del jaguar y sus implicaciones para la conservación en la Península de Yucatán, Análisis de viabilidad de poblaciones y hábitat del jaguar en México. In G. Ceballos, C. Chávez, R. List, \& H. Zarza (Eds.), Conservación y Manejo del Jaguar en México estudios de caso y Perspectivas (pp. 101-110). México: Alianza WWF/telcel, CONABIO, CONANP, EcoCiencias S.C.

Díaz-Pulido, A. \& Payán, E. (2011). Densidad de ocelotes (Leopardus pardalis) en los llanos colombianos. Mastozoología Neotropical, 18(1), 63-71.

Di Bitetti, M., Paviolo, A., \& De Angelo, C. (2006). Density, hábitat use and activity patterns of ocelots (Leopardus pardalis) in Atlantic Forest of Misiones, Argentina. Journal of Zoology, 270, 153-163.

Dillon, A., \& Kelly, J. M. (2008). Ocelot home range, overlap and density: comparing radio telemetry with camera trapping. Journal of Zoology, 275(4), 391-398.

Dirzo, R., Young, H. S., Galetti, M., Ceballos, G., Isaac, N. J. B., \& Collen, B. (2014). Defaunation in the Anthropocene. Science, 345, 401-406.

ESRI. (1994). Arc View versión 3.2. New York: Enviromental Systems Reserch Institute.

Estes, J. A., Terborgh, J., Brashares, J. S., Power, M. E., Berger, J., Bond, W. J., ... Wardle, D. A. (2011). Trophic Downgrading of Planet Earth. Science, 333, 301-306.

Faller, J. C. (2007). El Área Natural Protegida El Zapotal Conservación privada en Yucatán. Biodiversitas, 75, 1-7.

Faller, J. C., Chávez, C., Johnson, S., \& Ceballos, G. (2007). Densidad de la población de jaguares en el Noreste de la Península de Yucatán. In G. Ceballos, C. Chávez, R. List, \& H. Zarza (Eds.), Conservación y Manejo del Jaguar en México estudios de caso y Perspectivas (pp. 111-121). México: Alianza WWF/telcel, CONABIO, CONANP, EcoCiencias S.C. México.

Fisher, J., \& Wilkinson, L. (2005). The response of mammals to forest fire and timber harvest in the North America boreal forest. Mammal Review, 35(1), 51-81.

Gómez-Pompa, A., Allen, M. F., Fedick, S., \& JiménezOsorio, J. J. (2003). The Lowland Maya Area. Threee Millennia at the Human-Wildland Interface Binghamton. New York: The Haworth Press.

Gómez-Pompa, A., Lazcano-Barrero, M. A., GómezBarrero, A., \& Macswiney, C. (2011). La Reserva Ecológica El Edén: Proyecto de Conservación Privada. In J. Carabias, J. Sarukhán, J. de la Maza, \& C. Galindo (Coord.). México: Patrimonio Natural de 
México: Cien Casos de Éxito (pp. 92-93). México: CONABIO.

Gopalaswamy, A. M., Royle, J. A., Hines, J. E., Singh, P., Jathanna, D., Samba-Kumar, N., \& Karanth, U. (2012). Program SPACECAP: software for estimating animal density using spatially explicit capturerecapture models. Methods in Ecology and Evolution, 3, 1067-1072.

Hoogesteijn, R., Hoogesteijn, A., \& Mondolfi, E. (1993). Jaguar predation and conservation: cattle mortality caused by felines on three ranches in the Venezuelan llanos. In N. Dunstone, \& M. L. Gorman (Eds.), Mammals as predators (pp. 391-407). London: Zoological Society.

Karanth, K. U., \& Nichols, J. D. (1998). Estimation of Tiger densities in India using photographic captures and recaptures. Ecology, 79, 2852-2862.

Karanth, K. U., \& Nichols, J. D. (2000). Ecological status and conservation of tigers in India (Final Technical Report). Division of International Conservation, U.S. Fish and Wildlife Service, Wildlife Conservation Society. Bangalore, India: Centre for Wildlife Studies.

Karanth, K. U., \& Nichols, J. D. (2002). Monitoring tigers and their prey: A manual for researchers, managers and conservation Society. New York: Centre for Wildlife Studies, Bangalore, India

Kelly, M. J. (2003). Jaguar monitoring in Western Belize. Caribbean Geography, 13, 19-32.

Kelly, M., Noss, A., Di Bitetti, M., Maffei, L., Arispe, R., Paviolo, A., ... Di Blanco, Y. (2008). Estimating puma densities from camera trapping across three study sites: Bolivia, Argentina and Belize. Journal of Mammalogy, 89, 408-418.

Lazcano-Barrero, M. A., March, J., Núñez, H., Ruelas E., Muñoz-Alonso, A., \& Martínez, R. (1992). Inventario faunistico de la Reserva El Edén, Quintana Roo: Una prospección. ECOSFERA A. C. (Reporte Técnico). San Cristóbal de Las Casas, México.

Maffei, L., Cuellar, E., \& Noss, A. (2002). Uso de trampascámara para la evaluación de mamíferos en el ecotono Chaco-Chiquitanía. Revista Boliviana de Ecología y Conservación Ambiental, 11, 55-65.

Maffei, L., Cuellar, E., \& Noss, A. (2004). One thousand jaguars (Panthera onca) in Bolivia's Chaco? Camara Trapping in the Kaa-Iya National Park. Journal of Zoology, 262, 295-304.

Maffei, L., Noss, A., \& Fiorello, C. (2007). The jaguarundi (Puma yagouaroundi) in the Kaa-Iya del Gran Chaco National Park, Santa Cruz Bolivia. Mastozoología Neotropical, 14(2), 263-266.

Manterola, C., Conde, D. A., Colchero, F., Rivera, A., Huerta, E., Soler, A., \& Pallares, E. (2011). El jaguar como elemento estratégico para la conservación.
México: Colección Corredor Biológico Mesoamericano, CONABIO

Michalski, F., \& Peres, C. A. (2005). Anthropogenic determinants of primate and carnivore local extinctions in a fragmented forest landscape of southern Amazonia. Biological Conservation, 124, 383-396.

Miller, C., \& Miller, B. (2005). Jaguar Density in la Selva Maya (Informe Técnico no publicado). Belice: Wildlife Conservation Society.

Monroy-Vilchis, O., Zarco-González, M., Rodríguez-Soto, C., Soria-Díaz, L., \& Urios, V. (2011). Fototrampeo de mamíferos en la Sierra de Nanchititla, México: abundancia relativa y patrón de actividad. Revista Biología Tropical, 59(1), 373-383.

Navarro, C. J., Ramolina J. F., \& Pérez, J. J. (2007). El jaguar en Yum Balam y el Norte de Quintana Roo. In G. Ceballos, C. Chávez, R. List, \& H. Zarza (Eds.), Conservación y manejo del jaguar en México: estudio de caso y perspectivas (pp. 123-132). México: CONABIO, Alianza WWF, Telcel, Universidad Nacional Autónoma de México.

Noss, A., Kelly, M., Camblos, H., \& Ruiz, D. (2004). Pumas y jaguares simpátricos: datos de trampa cámara en Bolivia y Belize. VI Conferencia Internacional para el Manejo de Fauna silvestre en Amazonia y Latinoamerica (pp. 229-237). Iquitos, Perú: Universidad Nacional de la Amazonia Peruana.

Noss, A., Gardner, B., Maffei, L., Cuéllar, E., Montaño, A., Romero-Muñoz, R., ... O'Conell, A. F. (2012). Comparison of density estimation methods for mammal population with camera traps in the Kaa-Iya del Gran Chaco landscape. Animal Conservation, 15, 527-535.

Otis, D. L., Burnham, K. P., White, G. C., \& Anderson, D. R. (1978). Statistical inference from capture data on closed animal populations. Wildlife Monographs, $62,1-135$.

Pimm, S. L., Jenkins, C. N., Abell, R., Brooks, T. M., Gittleman, J. K., Joppa, L. N., ... Sexto, J. O. (2014). The Biodiversity of species and their rates of extinction, distribution, and Protection. Science, 344(6187), 987-998.

Rexstad, E., \& Burnham, K. P. (1991). Users Guide for Interactive Program CAPTURE. Abundance Estimation of Closed Populations. USA: Colorado State University, Fort Collins, Colorado.

Rodríguez-Reynaga, F., García-Contreras, G., Durán, R., Andrade, M., Merediz, G., Bermúdez, D., \& Franquesa, A. (2007). Plan de Conservación de Áreas. Zona Noreste de la Península de Yucatán. México: CICY, TNC, ASK, PPY.

Rosas-Rosas, O., \& Bender, G. (2012). Population Status of Jaguar (Panthera onca) y pumas (Puma concolor) 
in Northeastern Sonora, Mexico. Acta Zoológica Mexicana, 28(1), 86-101.

Salom, R., Carillo, E., Sáenz, J. C., \& Mora, J. (2007). Critical condition of jaguar Panthera onca population in Cocovado National Park, Costa Rica. Oryx, 41(1), 51-56.

Sánchez, A. R. \& Rebollar, S. (1999). Deforestación en la Península de Yucatán, los Retos que enfrentar. Madera y Bosques, 5(2), 3-17.

Sanderson, E., Redford, K. Chetkiewicz, C. Medellin, R., Rabinowitz, A., Robinson, J., \& Taber, A. (2002). Planning to Save a Species: the jaguar as a Model. Conservation Biology, 16(1), 58-72.

Sarukhán, J., Carabias, J., Koleff, P., \& Urquiza-Haas, T. (2012). Capital Natural de México: Acciones Estratégicas para su valoración, preservación y recuperación. México: CONABIO.

Sedesol (Secretaria de Desarrollo Social). (1994). Norma Oficial Mexicana. NOM-059-ECOL-1994. Diario Oficial de la Federación. Tomo CDLXXXVIII, (10), $2-60$.

Semarnat. (2002). Programa de Manejo Reserva de la Biosfera Ría Celestún. México: Secretaria de Medio Ambiente y Recursos Naturales.

Semarnat. (2010). Norma Oficial Mexicana NOM-059ECOL-2010. Protección ambiental-especies nativas de México de flora y fauna silvestres. Categorías de riesgo y especificaciones para su inclusión, exclusión o cambio. Lista de especies en riesgo. México. Diario Oficial de la Federación, 30 de Diciembre de 2010.
Silver, S. (2004). Assesing jaguar abundance using remotely triggered cameras. Jaguar Conservation Programs. USA: Wildlife Conservation Society.

Silver, S. C., Ostro, L. E. T., Marsh, L. K., Maffei, L., Kelly, J. M., Wallace, R. B., ... Ayala, G. (2004). The use of camera traps for estimating jaguar Panthera onca abundandace and density using capture/recapture analysis. Oryx, 38, 148-154.

Sosa-Escalante, J. E., Pech-Canché, J. M., MacSwiney, M. C., \& Hernández-Betancourt, S. (2013). Mamíferos terrestres de la Península de Yucatán, México, endemismo y riesgo. Revista Mexicana de Biodiversidad, 84.

Tello, J. L. (1986). The situation of wild cats (Felidae) in Bolivia. Switzerland: CITES Report.

Tobler, M., Carrillo-Percastegui, M., Zúñiga, H. A., \& Powell, G. (2013). High jaguar densities and large population sizes in the core habitat of the southwestern Amazon. Biological Conservation, 159, 375-381.

Tobler, M., \& Powell, G. (2013). Estimating jaguar densities with camera traps: Problems with current designs and recommendations for future studies. Biological Conservation, 129, 109-118.

Torres-Romero, E. J. (2009). Densidad, abundancia, uso de hábitat y patrones de actividad del ocelote (Leopardus pardalis) en la zona noreste de Quintana Roo: Estudio usando cámaras trampa (Tesis de maestría). México: El Colegio de la Frontera Sur.

Yasuda, M. (2004). Monitoring diversity and abundance of mammals with camera traps: a case study on Mount Tsukuba, central Japan. Mammal Study, 29, 27-46. 
